\title{
The Roles of Cohesive Strength and Toughness for Crack Growth in Visco-elastic and Creeping Materials
}

\author{
H. Wang ${ }^{\dagger}$, W. Lu ${ }^{\dagger}$, J. R. Barber ${ }^{\dagger}$ and M. D. Thouless ${ }^{\dagger, \dagger}$ \\ ${ }^{\dagger}$ Department of Mechanical Engineering \\ $\ddagger$ Department of Materials Science \& Engineering \\ University of Michigan, Ann Arbor, MI 48109, USA
}

\begin{abstract}
A cohesive-zone analysis for crack propagation in a linear viscous / creeping material, with rate-independent fracture parameters, is presented. The analysis is done for a double-cantilever beam geometry, and compared to an analytical solution for a creeping beam on an elastic foundation. It is shown that there are two regimes for viscous crack growth. One regime occurs in the limit of small viscous fracture lengths, when the crack-tip stress field has the inverse square-root dependence expected in the limit of classical fracture mechanics that does not have a fracture length. In this regime, the crack velocity is proportional to $K^{4}$, where $K$ is the stress-intensity factor. The second regime occurs for large viscous fracture lengths, where classical fracture mechanics is not appropriate. In this regime, the crack velocity has a weaker dependence on the applied load, and can be modelled accurately by the solution to the elastic-foundation problem. The expected transition from viscous behavior to elastic fracture at higher velocities is observed from the numerical simulations, when the viscous fracture length becomes significantly greater than the elastic fracture length.
\end{abstract}

October 1, 2015 


\section{Introduction}

Modeling crack growth requires an understanding of which material parameters control fracture. For example, linear-elastic fracture mechanics (LEFM) indicates that the fracture load is controlled by the modulus, $E$, the toughness, $\Gamma$, and a characteristic length of the geometry, $h$. However, there are many situations where a second fracture parameter, such as the cohesive strength, $\hat{\sigma}$, is required. Alternative approaches use concepts such as critical displacements or micro-structural lengths. The common feature is that some form of characteristic length scale, whether a physical length or a group of fracture parameters with dimensions of length, such as $E \Gamma / \hat{\sigma}^{2}$, needs to be introduced into the formulation.

The motivation for this study was to determine the fracture parameters that control crack growth in a creeping solid. We addressed this by conducting a cohesive-zone analysis with a linear visco-elastic material. Here, we show that, in contrast to fracture controlled by an elastic stress field, there are no conditions under which crack growth can be modelled in a viscous or creeping material, without introducing a fracture length of some description. This is true, even in regimes where the crack-tip stresses follow the continuum singular field.

Time-dependent crack growth has historically been studied in two distinct areas

of research: creep rupture of metals and ceramics, and fracture of polymers. These two areas have developed two distinct frameworks, with different implications, for what is essentially the same problem. The creep-rupture literature tends to describe the problem in terms of how the time-dependent properties of a material result in sub-critical cracking at low driving forces (an apparent weakening). The polymers lit- 
erature tends to describe the problem in terms of how the time-dependent properties of a material result in an increased rate of energy dissipation (an apparent increase in toughness). This is a manifestation of the classic contrast between strength and toughness.

Creep-crack growth models are often formulated in terms of the nucleation and growth of cavities ahead of a crack tip $[1,2,3]$. This cavity growth can be linked to the deformation of the surrounding material, assuming that the damage is embedded within the crack-tip stress field appropriate for a creeping solid $[4,5,6,7]$. These analyses introduce a characteristic length scale into the problem for dimensional consistency. For example, in the model of Cocks and Ashby [4], this length scale is the distance over which the damage is assumed to grow under the influence of the cracktip stress field.

The predictions for the crack velocity depend on assumptions about how the damage interacts with the stress field, and how the elastic and creep stresses evolve at the crack tip. However, the different models share a common aspect in that the crack velocity depends on the crack-tip loading parameter for creep, $C^{*}$, which is the time-dependent analogue of the $J$-integral $[8,9]$. The effect of creep / viscosity is to cause sub-critical crack growth until the cracks are long enough to cause catastrophic failure. The implication of this view-point is that viscosity weakens a material, since it can provide a mechanism for pre-existing damage to grow.

The physics of time-dependent fracture of polymers is essentially identical to that of creep rupture. However, the literature often focuses on the concept of a ratedependent toughness $[10,11,12]$, rather than on how the crack velocity varies with 
driving force. The viscous energy dissipated at the crack tip is seen as contributing to the toughness, and the size of the crack-tip viscous zone depends on the crack velocity [13]

Cohesive-zone models of fracture automatically provide such a suitable length scale to describe creep crack growth through the ratio of the toughness to the cohesive strength. The concept of cohesive tractions has been used in the polymers literature for a very long time [14]. Numerical analyses of time-dependent fracture using cohesive-zone elements have been developed for time-dependent [15] and timeindependent [16] cohesive models. In this work, we explore crack growth using a time-independent cohesive-zone model embedded in a visco-elastic material. This is essentially identical to the assumptions made in the analysis by Rahulkumar et al. [16] in their analysis of the peel test. However, since we approached the problem from a creep-rupture perspective, not from the rate-dependent toughness perspective of the polymer literature, our results give a different insight (although they are consistent with the earlier work). Furthermore, by using the simple geometry of a moment-loaded double-cantilever beam, we avoided the complication common to many geometries that the cohesive strength affects the conditions for elastic crack propagation. This permitted a clean relationship between the crack-growth rate and the cohesive parameters to be developed.

\section{Beam Analysis}

An analytical approximation for a cohesive-zone model with a linear traction-separation law of a double-cantilever beam (DCB) subjected to an applied moment of $M_{\infty}$ (per 
unit width) can be obtained from the solution for a beam (of unit width) on an elastic foundation (Fig. 1). Owing to symmetry and the resultant pure mode-I conditions, only one arm is considered. It is assumed that the springs are linear elastic with a spring constant $k$, so that they exert tractions along the beam of

$$
T_{n}=-k v
$$

where $v$ is the displacement of the beam. Failure of the spring occurs when its extension reaches a critical value, so that in terms of the usual parameters for a cohesive zone, the spring constant can be expressed as

$$
k=\hat{\sigma}^{2} / \Gamma
$$

where $\hat{\sigma}$ is the cohesive strength, $\Gamma$ is the toughness (recognizing that there are two halves to the DCB geometry).

\subsection{Elastic analysis}

If the beams are elastic, the problem and solution are well-known. The governing equation is

$$
\frac{E h^{3}}{12} \frac{d^{4} v}{d z^{4}}+\frac{\hat{\sigma}^{2}}{\Gamma} v=0 .
$$

For which a solution is given by Barber [17] as

$$
\tilde{v}=\frac{2 \beta_{o}^{2} M_{\infty} \Gamma}{\hat{\sigma}^{2} h^{3}} \exp ^{-\beta_{o} \tilde{z}}\left(\cos \beta_{o} \tilde{z}-\sin \beta_{o} \tilde{z}\right)
$$

In this equation, $\tilde{v}=v / h, \tilde{z}=z / h$, and

$$
\beta_{o}=\left(\frac{3 k h}{E}\right)^{1 / 4}=\sqrt[4]{3} \tilde{\zeta}^{-1 / 4},
$$

where $\zeta=E \Gamma / \hat{\sigma}^{2}$ is the elastic fracture length (for plane stress), and $\tilde{\zeta}=\zeta / h$ is the corresponding elastic fracture-length scale [20]. 
The stresses ahead of the crack can be calculated from $\sigma(\tilde{z})=-T_{n}(\tilde{z})$, which from Eqn. 1 is given by

$$
\frac{\sigma(\tilde{z})}{\hat{\sigma}}=\frac{k h \tilde{v}(\tilde{z})}{\hat{\sigma}}=\frac{\hat{\sigma} h}{\Gamma} \tilde{v}(\tilde{z}) .
$$

The condition for crack propagation can be found by equating $\sigma(0) / \hat{\sigma}$ to 1 at the crack tip, and substituting the resulting value of $\tilde{v}(0)=\Gamma / \hat{\sigma} h$ into Eqn. 4 :

$$
\frac{M_{\infty}}{\Gamma h}=\frac{1}{\sqrt{12}}\left(\frac{E h}{\Gamma}\right)^{1 / 2} .
$$

This is the well-known result that the fracture conditions for a DCB loaded by a pure moment is independent of the interfacial cohesive law (unlike the case where the DCB is loaded by a point load [18]).

\subsection{Viscous analysis}

Using the correspondence principle, this analysis can be repeated for a linear-viscous beam. The deformation is related to the local bending moment, $M(z)$ by

$$
\frac{\partial^{3} v}{\partial z^{2} \partial t}=-\frac{12 M(z)}{\eta h^{3}}
$$

where $\eta$ is the uniaxial viscosity, and $h$ is the thickness of the beam. The moment is related to the tractions by

$$
\frac{d^{2} M(z)}{d z^{2}}=-T_{n}(z)=k v(z)
$$

If the crack is propagating at a constant velocity of $\dot{a}$, so that there is steady-state, a new non-dimensional co-ordinate can be defined as $\tilde{z}=(z-\dot{a} t) / h$. Using the nondimensional displacement, $\tilde{v}=v / h$, Eqns. 7 and 8 can be combined to obtain the steady-state governing equation for a linear-viscous DCB:

$$
\frac{d^{5} \tilde{v}(\tilde{z})}{d \tilde{z}^{5}}-\frac{12 k h^{2}}{\eta \dot{a}} \tilde{v}=0 .
$$


The solution to this equation is of the form

$$
\tilde{v}(\tilde{z})=A \exp ^{b \tilde{z}}
$$

where

$$
b=[ \pm \cos (\pi / 5) \pm i \sin (\pi / 5)] \lambda_{o}, b=\lambda_{o}
$$

and

$$
\lambda_{o}=\left(\frac{12 k h^{2}}{\eta \dot{a}}\right)^{1 / 5}=\left(\frac{12 \hat{\sigma}^{2} h^{2}}{\eta \Gamma \dot{a}}\right)^{1 / 5}
$$

However, only the two roots with a negative real component can be a physical solution, which can be written as

$$
\tilde{v}(\tilde{z})=\exp ^{-\lambda_{o} \tilde{z} \cos (\pi / 5)}\left\{B_{1} \cos \left[\lambda_{o} \tilde{z} \sin (\pi / 5)\right]+B_{2} \sin \left[\lambda_{o} \tilde{z} \sin (\pi / 5)\right]\right\}
$$

The two boundary conditions for this problem are (i) the moment at the crack tip is always equal to the applied moment, so that $M(0)=-M_{\infty}$, and (ii) the shear force at the crack tip is 0 . Therefore,

$$
\begin{array}{r}
\left.\frac{d^{3} \tilde{v}}{d \tilde{z}^{3}}\right|_{\tilde{z}=0}=\frac{12 M_{\infty}}{\eta h \dot{a}} \\
\left.\frac{d^{4} \tilde{v}}{d \tilde{z}^{4}}\right|_{\tilde{z}=0}=0 .
\end{array}
$$

With these boundary conditions, the solution to the crack propagation problem is

$$
\tilde{v}(\tilde{z})=\frac{M_{\infty}}{\lambda_{o}^{3} \eta h \dot{a}} \exp ^{-\lambda_{o} \tilde{z} \cos (\pi / 5)}\left\{-12 \cos \left[\lambda_{o} \tilde{z} \sin (\pi / 5)\right]+16.5 \sin \left[\lambda_{o} \tilde{z} \sin (\pi / 5)\right]\right\}
$$

As described above for the elastic case, the stresses ahead of the crack can be found from the traction distribution, and are given by

$$
\frac{\sigma(\tilde{z})}{\hat{\sigma}}=\frac{k h \tilde{v}(\tilde{z})}{\hat{\sigma}}=\frac{\hat{\sigma} h}{\Gamma} \tilde{v}(\tilde{z})
$$


The steady-state crack velocity can be found by noting that $\sigma(0) / \hat{\sigma}=1$ at the crack tip, and substituting the resulting value of $\tilde{v}(0)=\Gamma / \hat{\sigma} h$ into Eqn. 13:

$$
\frac{\dot{a} \eta \Gamma}{\hat{\sigma}^{2} h^{2}}=12\left(\frac{M_{\infty}}{\hat{\sigma} h^{2}}\right)^{2.5} .
$$

The term on the left-hand side is the viscous fracture-length scale, $\tilde{\zeta}_{v}=\zeta_{v} / h$, which is the analogue of the elastic fracture-length scale, $\tilde{\zeta}$, defined earlier ${ }^{1}$.

\section{Cohesive-zone analysis}

Numerical simulations were conducted using the commercial finite-element package ABAQUS, with the cohesive-zone elements defined through a user-defined subroutine [19]. This cohesive-zone model is a mixed-mode formulation; however, the symmetry conditions of the DCB geometry ensured that this particular study was pure mode-I. The traction-separation law was chosen to be rate-independent, and of the form given by Eqn. 1. (A steep linear decay occurred after the cohesive strength had been reached; the work corresponding to this portion was $0.5 \%$ of the work done during the rising portion of the curve.) The cohesive-zone was given a finite thickness, $d$, corresponding to $d / h=6.7 \times 10^{-4}$.

The constitutive properties of the arms were defined through the ABAQUS subroutine CREEP as

$$
\dot{\epsilon}_{i j}=\frac{(1+\nu) \dot{\sigma}_{i j}}{E}-\frac{\nu}{E} \dot{\sigma}_{k k} \delta_{i j}+\frac{3 \sigma_{i j}^{\prime}}{2 \eta},
$$

(using standard tensor notation), where $\eta$ is the uniaxial viscosity, and $\sigma_{i j}^{\prime}$ is the deviatoric stress. So, a Maxwell type of material was studied, representing creep, rather

\footnotetext{
${ }^{1} \mathrm{~A}$ comparison between the elastic and viscous fracture lengths suggests a plane-stress viscoelastic definition would be given by $\Gamma E\left(1-\exp ^{-\dot{a} \eta / E h}\right) / \hat{\sigma}^{2} h$.
} 
than a standard-linear solid with a non-zero fully-relaxed modulus, representing a polymer. In the numerical calculations that follow, $\nu$ was set to 0.49999 .

The geometry for the finite-element calculations is shown in Fig. 2. The loading couples were applied as a linear distribution of tractions to one end of each beam. The other end of each beam was clamped. The calculations were implicit, with the elements being first-order, coupled temperature-displacement, plane-stress elements, with reduced integration points (CPS4RT). To ensure accurate numerical results, the size, $l_{o}$, of the smallest element at the tip of the crack needs to be much smaller than the elastic fracture length [20]. In the present case, $l_{o}$ was limited to be no larger than $10^{-4} E \Gamma / \hat{\sigma}^{2}$. At the lowest crack velocities, this resulted in a mesh size that could be as high as ten times the viscous fracture length. However, a mesh-sensitivity analysis showed that, even in this range, the results were mesh insensitive within the limits of the error bars shown in the figures.

The visco-elastic calculations were done by applying a constant moment. There was an incubation period before the crack started growing. After a very short transition, the crack reached a steady-state velocity which was measured. The time increment for the calculations, $\Delta t$, was set to satisfy the condition $\dot{a} \Delta t / l_{o}<50$. Within this limit there was no significant sensitivity of the results to the time increment.

\section{Results}

A dimensional analysis for the problem of a crack growing at the interface between two linear, creeping beams shows that the steady-state crack velocity must be of the 
form

$$
\frac{\dot{a} \eta}{\Gamma}=f\left(\frac{E h}{\Gamma}, \frac{\Gamma}{\hat{\sigma} h}, \frac{M_{\infty}}{\Gamma h}\right) .
$$

Figure 3 is calculated from the cohesive-zone analysis, and shows how the crack velocity varies with applied moment for different levels of the cohesive strength.

It will be noted that the results in Fig. 3 exhibit an asymptotic limit, given by Eqn. 6, where the crack velocity tends to infinity when the condition for elastic fracture is met. At lower levels of the applied moment, in the viscous dominated regime where the crack velocity is lower, there is a power-law relationship between the crack velocity and the applied moment. The conditions for this viscous limit to be valid is that $\dot{a} \eta / E h<<1$. This non-dimensional group is equal to the ratio between the viscous and elastic fracture lengths. So, viscous crack growth occurs when $\zeta_{v}<<\zeta$. However, it is immediately obvious from Fig. 3 that the power-law relationship describing the relationship between crack velocity and applied moment is not unique, and it only matches the analytical solution given in Eqn. 15 for the larger values of $\Gamma / \hat{\sigma} h$.

A detailed exploration of the viscous crack velocity in non-dimensional space shows that it can always be expressed simply as

$$
\frac{\dot{a} \eta \Gamma}{\hat{\sigma}^{2} h^{2}}=f\left(\frac{M_{\infty}}{\hat{\sigma} h^{2}}\right)
$$

in the limit where $\dot{a} \eta / E h<<1$. This is shown in Fig. 4. It will be seen that the analytical beam result of Eqn. 15 is valid for large viscous fracture-length scales. For smaller viscous fracture-length scales, an empirical fit to the data suggests that

$$
\frac{\dot{a} \eta \Gamma}{\hat{\sigma}^{2} h^{2}} \approx 70\left(\frac{M_{\infty}}{\hat{\sigma} h^{2}}\right)^{4} .
$$


The fact that there are two regimes of behavior, depending on the fracture-length scale, is very reminiscent of what is seen with elastic fracture. In elastic fracture, small fracture-length scales correspond to a regime of toughness-controlled fracture (with an inverse square-root singular stress field near the crack tip), large fracture-length scales correspond to a regime of strength-controlled fracture. The particular geometry of a moment-loaded DCB is a special case for which the elastic fracture strength depends only on toughness; it doesn't change with the elastic fracture-length scale, $\tilde{\zeta}$. As a result, the analytical beam solution always gives the correct result for the critical moment required to propagate a crack. However, as shown in Fig. 5, the ability of this analytical solution to give the correct stress field depends on $\tilde{\zeta}$. At small values of $\tilde{\zeta}$, the beam analysis does not capture details of the multi-dimensional stress field and the inverse-singular field expected from linear-elastic fracture mechanics (LEFM). As demonstrated elsewhere [21, 22], a cohesive-zone analysis does capture the inverse-square root field, over an intermediate distance from the crack tip, provided $\tilde{\zeta}$ is small enough. Conversely, the $K$-field of LEFM does not provide any form of approximation to the stress field at large values of $\tilde{\zeta}$, while the beam analysis provides a good description of the stress field under these conditions (Fig. 5).

A similar result, that the viscous-beam solution provides a good description of the stress field ahead of a crack for large values of the viscous fracture-length scale, $\tilde{\zeta}_{v}$, but does not do so for small values, can be seen from Fig. 6 . The inverse square-root singular field expected for a linear material is not captured by the beam solution when $\tilde{\zeta}_{v}$ is small, but it is captured by the cohesive-zone model. Conversely, when $\tilde{\zeta}_{v}$ is large, beam theory describes the stress field reasonably accurately. In contrast to the elastic DCB geometry, the conditions for crack growth in the viscous geometry 
depend on the fracture-length scale. As a result, beam theory doesn't describe the crack velocity correctly for small values of $\tilde{\zeta}_{v}$, but it does describe the crack velocity correctly for large values of $\tilde{\zeta}_{v}$.

\section{Discussion}

A summary of the three different regimes of creep-crack growth can be seen in Fig. 7 . In this particular geometry, the elastic fracture condition is given by the LEFM solution of Eqn. 6, irrespective of the fracture-length scale. Therefore, if

$$
\frac{M_{\infty}}{\hat{\sigma} h^{2}}>\frac{1}{\sqrt{12}}\left(\frac{E \Gamma}{\hat{\sigma}^{2} h}\right)^{1 / 2},
$$

there is no equilibrium solution, and the crack must propagate dynamically. As the applied bending moment approaches this limiting value, the crack velocity increases dramatically. If $M_{\infty} / \hat{\sigma} h^{2}$ is significantly below this value, then the crack velocity is given by either Eqn. 15 or Eqn. 19:

$$
\begin{aligned}
& \frac{\dot{a} \eta \Gamma}{\hat{\sigma}^{2} h^{2}}=12\left(\frac{M_{\infty}}{\hat{\sigma} h^{2}}\right)^{2.5}, \text { for } M_{\infty} / \hat{\sigma} h^{2}>0.35 \\
& \frac{\dot{a} \eta \Gamma}{\hat{\sigma}^{2} h^{2}} \approx 70\left(\frac{M_{\infty}}{\hat{\sigma} h^{2}}\right)^{4}, \text { for } M_{\infty} / \hat{\sigma} h^{2}<0.35 .
\end{aligned}
$$

Both of these two behaviors will only be observed for relatively tough materials, with large elastic fracture-length scales. As shown in Fig. 7, brittle materials may only exhibit the second type of behavior if the critical moment for elastic fracture is very low.

In the creep-rupture literature, the crack velocity is expressed in terms of $C^{*}$, which is equal to $K^{2} / \eta$. In the present case,

$$
C^{*}=12 M_{o}^{2} / \eta h^{3} .
$$


One would expect $C^{*}$ to be the controlling parameter, only when the stress field has an inverse square-root dependence; i.e., when the viscous fracture-length scale is small. Under these conditions, one can use the results of Eqn. 19 to express the crack velocity in terms of $C^{*}$ as

$$
\frac{\eta \dot{a}}{\Gamma}=0.05\left(\frac{\eta C^{*}}{\Gamma \hat{\sigma}}\right)^{2} .
$$

This formulation of the results reveals that the details of the geometry do not directly affect crack growth, except through $C^{*}$. This is expected in the limit of a $C^{*}$-dominated crack-growth, when the viscous fracture-length is small. However, it is interesting that, even in this regime, the dependence on $C^{*}$ is not the square-root dependence predicted by earlier creep-crack-growth models [4].

In the polymers literature, the crack velocity is related to the effective toughness, which includes a viscous dissipation term. The cohesive-zone analysis of Rahulkumar et al. [16] shows that the peak dissipation (and maximum effective toughness) occurs at an intermediate crack velocity. At high and low velocities, corresponding to the unrelaxed and relaxed elastic regimes, there is limited viscous dissipation and the effective toughness tends to the intrinsic toughness of the cohesive zone, $\Gamma$. A similar calculation for the viscous dissipation in the present model shows that the effective toughness increases without limit as the crack speed decreases. This is expected for a system in which the fully-relaxed modulus is zero.

In the light of this discussion, one can appreciate how a standard-linear solid might behave if approached from the vantage point of the present paper, rather than from the perspective of the polymers literature. The effect of a non-zero fully-relaxed 
modulus, $E_{r}$, would be to introduce a fourth regime. If

$$
\frac{M_{\infty}}{\hat{\sigma} h^{2}}<\frac{1}{\sqrt{12}}\left(\frac{E_{r} \Gamma}{\hat{\sigma}^{2} h}\right)^{1 / 2}
$$

there would be no crack growth. Above this value, either or both of the two regimes of viscous crack growth might be observed, before the upper limit associated with the unrelaxed modulus is reached. The details of the behavior would be expected to depend on the magnitudes of the fully-relaxed and unrelaxed elastic fracture-length scales. This appears to match the behavior described by Rahulkumar et al. [16] in their study, but is beyond the scope of the present paper.

\section{Conclusions}

A cohesive-zone analysis of linear viscous crack growth for a double-cantilever-beam geometry shows two distinct regimes of behavior. At low velocities, corresponding to a small viscous fracture length, when the crack-tip stress field is controlled by the stress-intensity factor, the crack velocity scales with $K^{4}$. At higher crack velocities, corresponding to higher viscous fracture lengths, the stress field can be modelled as a viscous beam on an elastic foundation. Under these conditions, there is no inverse square-root singular field, and the crack velocity grows with a much lower dependence on the applied loads. As the elastic fracture condition is approached, the crack velocity increases dramatically.

These results have been presented for a simple geometry that exhibits a steady state, and for which the elastic fracture condition is independent of the fracture-length scale. In more general geometries, the transition to the elastic fracture regime will be complicated because it may occur at lower loads if the elastic length scale is large. So, the nature of the visco-elastic crack growth may be complicated, with different types 
of growth rates depending on the viscous fracture length, and different transitions to elastic crack propagation depending on the elastic fracture length. It is further noted that a more general visco-elastic model, with a non-zero relaxed modulus, is expected to truncate the viscous crack growth at low velocities, providing a threshold.

These results have been presented in a creep-rupture format, emphasizing the sub-critical crack growth. From this perspective, viscosity serves to make a material weak, causing sub-critical crack growth. However, it is noted that the results are consistent with the common polymers perspective. The apparent toughness increases as the crack velocity decreases, owing to the increase in viscous dissipation. Rather than making the material weak, viscosity can be seen to make the material tougher. Obviously, this dichotomy in perspective from the two fields is a long-standing one, and the question of whether one might prefer a tough but weak material or a strong but brittle material depends on the application. However, the numerical crack-propagation model presented here, in which the crack velocity can be related to both the applied load and the energy dissipation, serves as a nice illustration of the issue.

\section{Acknowledgements}

This research was supported by the Consortium for Advanced Simulation of Light Water Reactors (http://www.casl.gov), an Energy Innovation Hub (http://www.energy.gov/hubs) for Modeling and Simulation of Nuclear Reactors under U.S. Department of Energy Contract No. DE-AC05-00OR22725. 


\section{References}

[1] D. S. Wilkinson and V. Vitek. The propagation of cracks by cavitation: a general theory. Acta Metallurgica, 30:1723-1732, 1982.

[2] J. L. Bassani and V. Vitek. Propagation of cracks under creep conditions. In Proceedings of 9th U.S. National Congress on Theoretical and Applied Mechanics, pages 127-133, 1982.

[3] M. D. Thouless, C. H. Hsueh and A. G. Evans. A damage model of creep crack growth in polycrystals. Acta Metallurgica, 31:1675-1687, 1983.

[4] A. C. F. Cocks and M. F. Ashby. The growth of a dominant crack in a creeping material. Scripta Metallurgica, 16:109-114, 1982.

[5] C. Y. Hui and V. Banthia. The extension of cracks at high temperature by growth and coalescence of voids. International Journal of Fracture, 25:53-67, 1984.

[6] H. Riedel. Creep crack growth under small-scale creep conditions. International Journal of Fracture, 42:173-188, 1990.

[7] J. L. Bassani and D. E. Hawk. Influence of damage on crack-tip fields under smallscale-creep conditions. International Journal of Fracture, 42:157-172, 1990.

[8] J. D. Landes and J. A. Begley. A fracture mechanics approach to creep crack growth. In J. R. Rice and P. C. Paris, editors, Mechanics of Materials, volume STP 590, pages 128-148. ASTM International, 1976.

[9] C. E. Turner K. M. Nikbin, G. A. Webster. Relevance of nonlinear fracture mechanics to creep cracking. In J. L. Swedlow and M. L. Williams, editors, Cracks and Fracture, volume STP 601, pages 47-62. ASTM International, 1976.

[10] E. H. Andrews and A. J. Kinloch. Mechanics of adhesive failure. I. Proceedings of the Royal Society of London, A332:385-399, 1973.

[11] R.-J. Chang and A. N. Gent. Effect of interfacial bonding on the strength of adhesion of elastomers. I Self-adhesion. Journal of Polymer Science, 19:1619$1633,1981$.

[12] J. G. Williams. Fracture Mechanics of Polymers. Ellis Horwood, Chichester, UK, 1984.

[13] D. B. Xu and C.-Y. Hui. Interface fracture and viscoelastic deformation in finite size specimens. Journal of Applied Physics, 72:3305-3316, 1992. 
[14] W. G. Knauss. Delayed failure - the Griffith problem for linearly viscoelastic materials. International Journal of Fracture Mechanics, 6:7-20, 1970.

[15] C. M. Landis, T. Pardoen and J. Hutchinson. Crack velocity dependent toughness in rate dependent materials. Mechanics of Materials, 32:663-678, 2000.

[16] P. Rahulkumar, A. Jagota, S. J. Bennison and S. Saigal. Cohesive element modeling of viscoelastic fracture: application to peel testing of polymers. International Journal of Solids and Structures, 37:1873-1897, 2000.

[17] J. R. Barber. Intermediate Mechanics of Materials. Springer, Dordrecht, The Netherlands, 2011.

[18] S. Li, J. Wang and M. D. Thouless. The effects of shear on delamination of beam-like geometries. Journal of the Mechanics and Physics of Solids, 52:193$214,2004$.

[19] Q. D. Yang and M. D. Thouless. Mixed-mode fracture analyses of plasticallydeforming adhesive joints. International Journal of Fracture, 110:175187, 2001.

[20] M. D. Thouless and R. B. Sills. The effect of cohesive-law parameters on mixedmode fracture. Engineering Fracture Mechanics, 109:353-368, 2013.

[21] J. P. Parmigiani and M. D. Thouless. The effects of cohesive strength and toughness on mixed-mode delamination of beam-like geometries. Engineering Fracture Mechanics, 74:2675-2699, 2007.

[22] M. D. Thouless and R. B. Sills. Cohesive-length scales for damage and toughening mechanisms. International Journal of Solids and Structures, 55:32-43, 2015. 


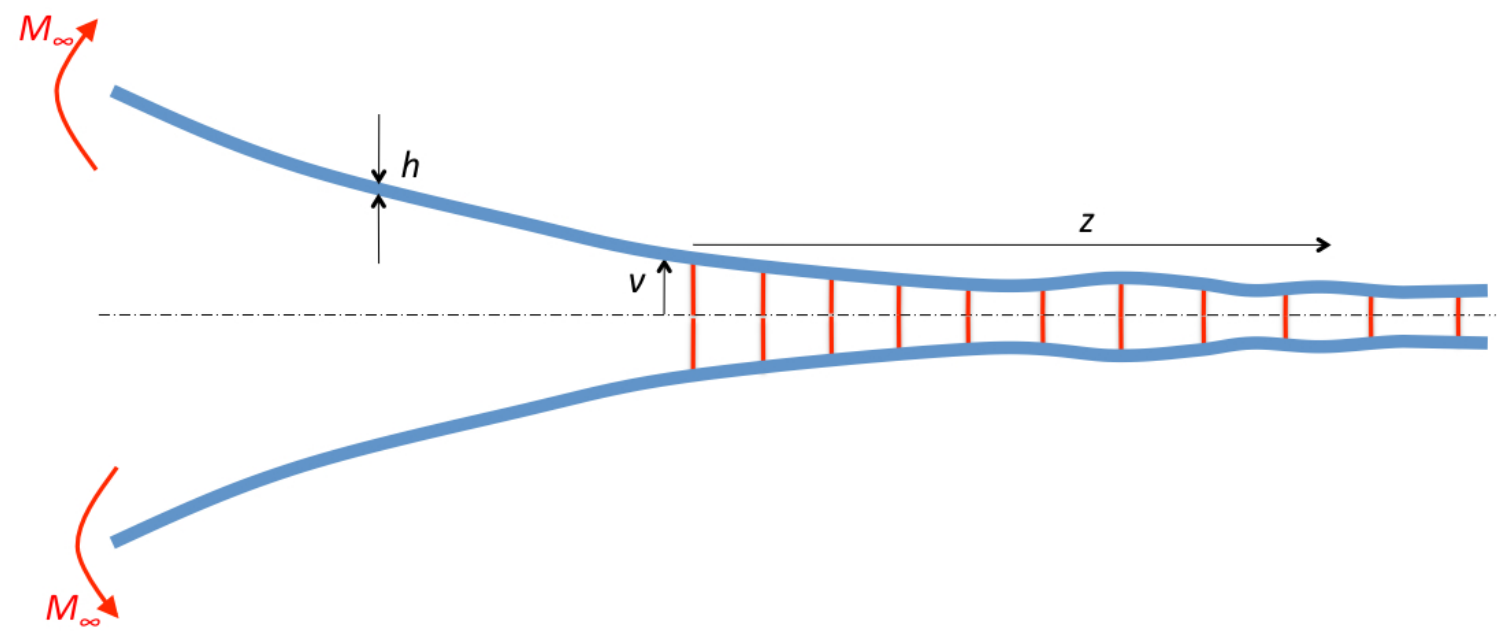

Figure 1: Geometry of a DCB with elastic springs along the interface 


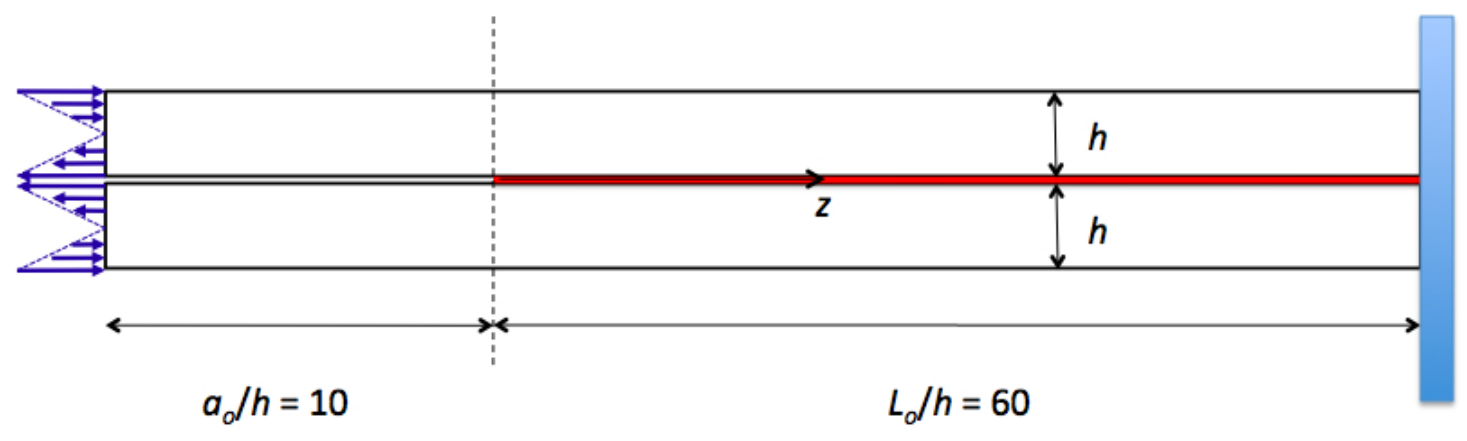

Figure 2: Geometry of a DCB used for the cohesive-zone analysis. 


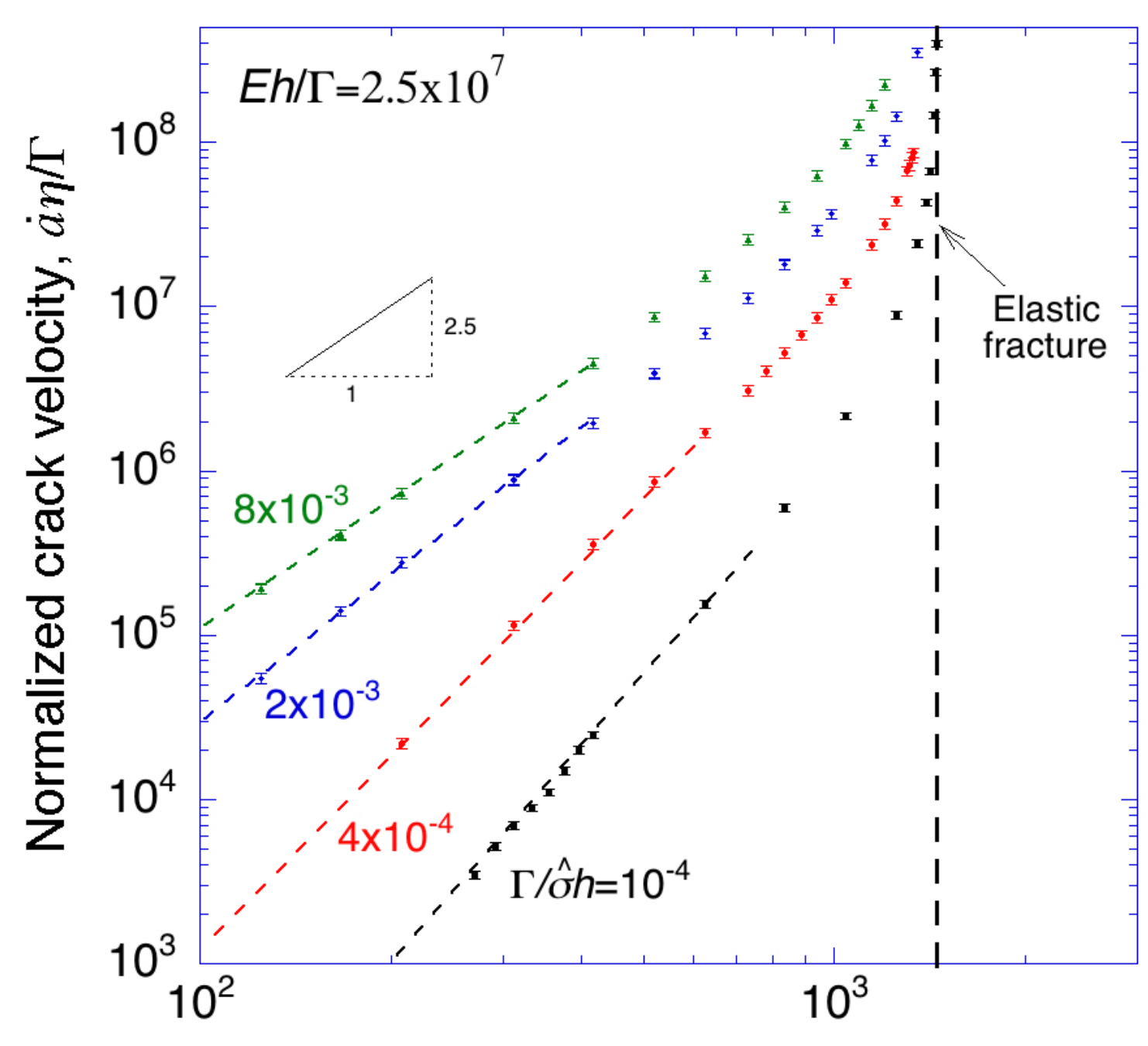

Normalized moment, $M_{\infty} / \Gamma h$

Figure 3: Non-dimensional crack velocity as a function of applied moment. As the magnitude of the applied moment approaches the value for elastic fracture, the crack velocity increases without limit. At lower values of the applied moment, the crack velocity depends only on the viscous properties of the DCB arms, not on the elastic properties. 


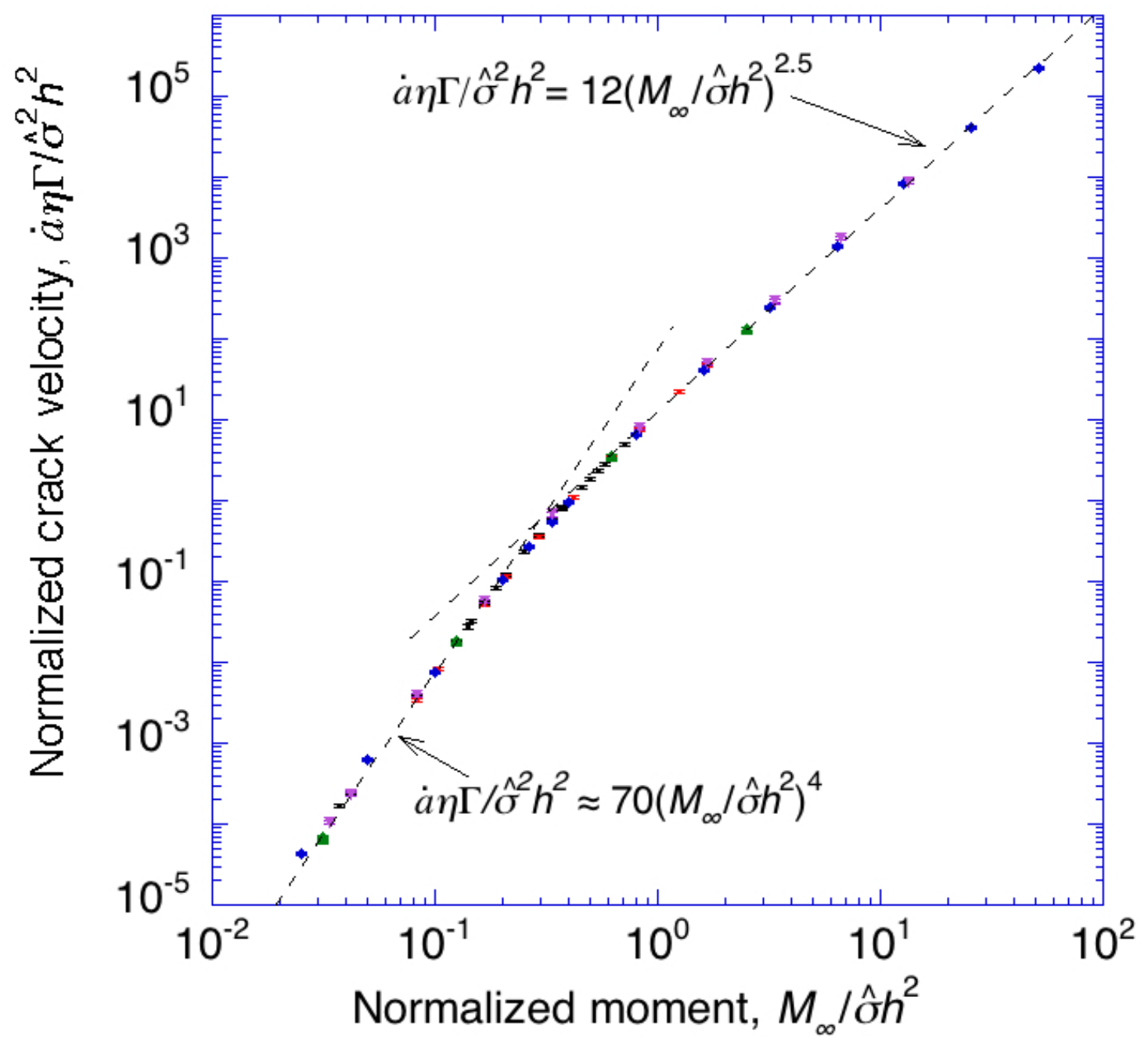

Figure 4: Non-dimensional crack velocity (viscous cohesive-length scale) as a function of applied moment in the viscous regime, showing two regimes of behavior at large and small cohesive-length scales. 

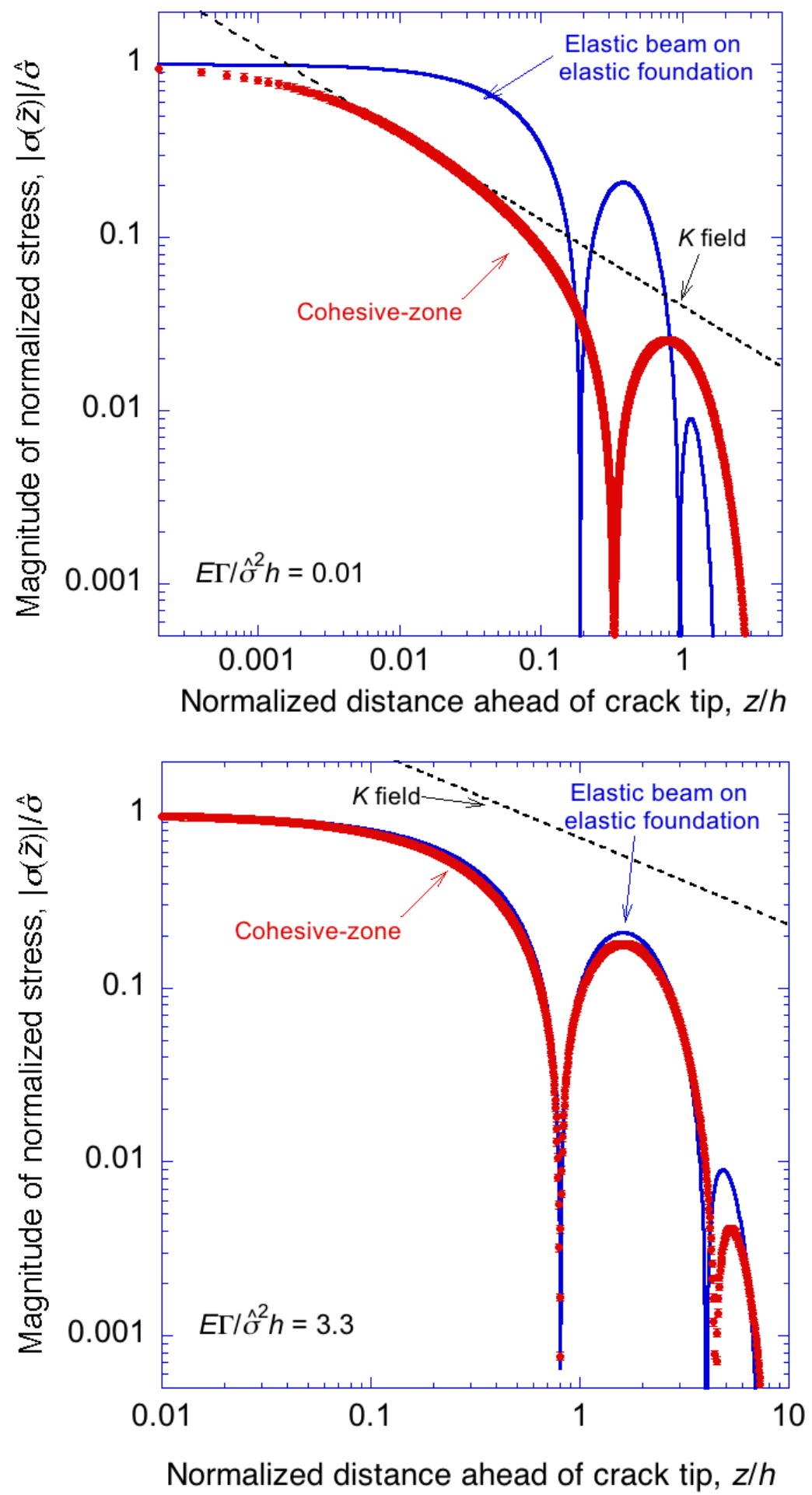

Figure 5: Comparison between stress distributions at fracture for an elastic doublecantilever beam using a cohesive-zone models and an elastic foundation model for a small and large cohesive-length scale. 


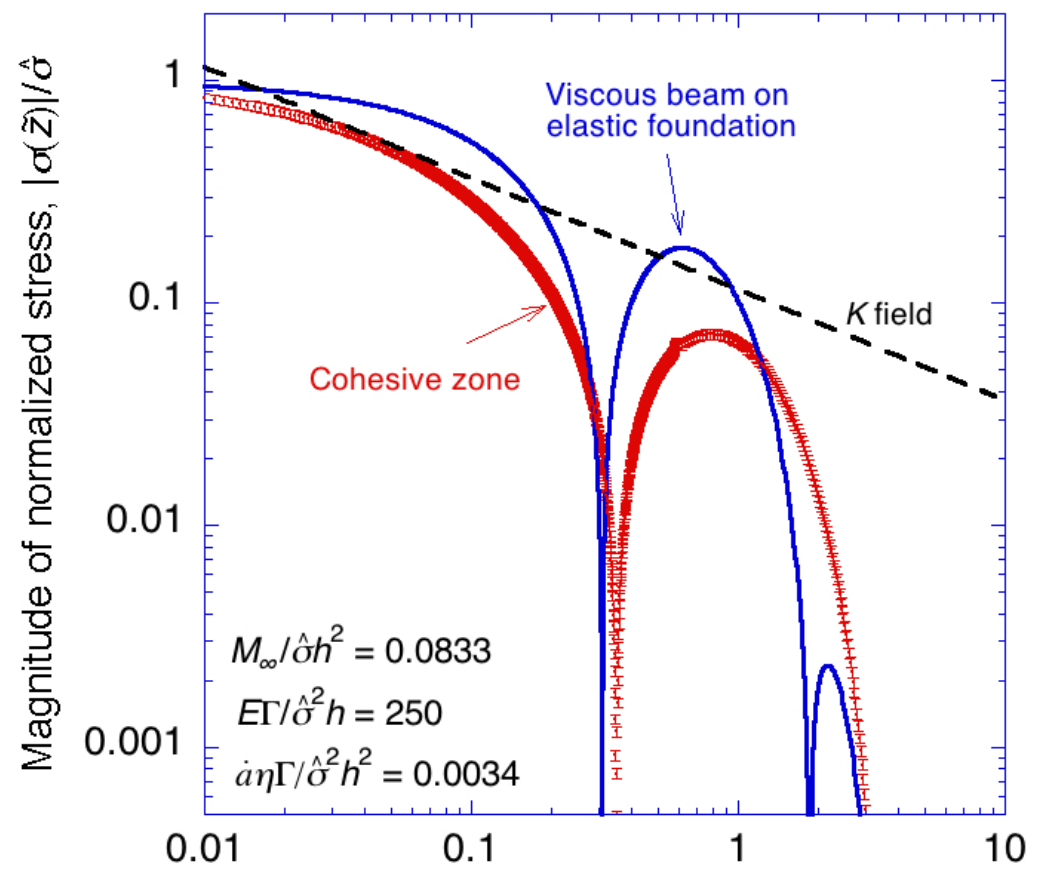

Normalized distance ahead of crack tip, $z / h$

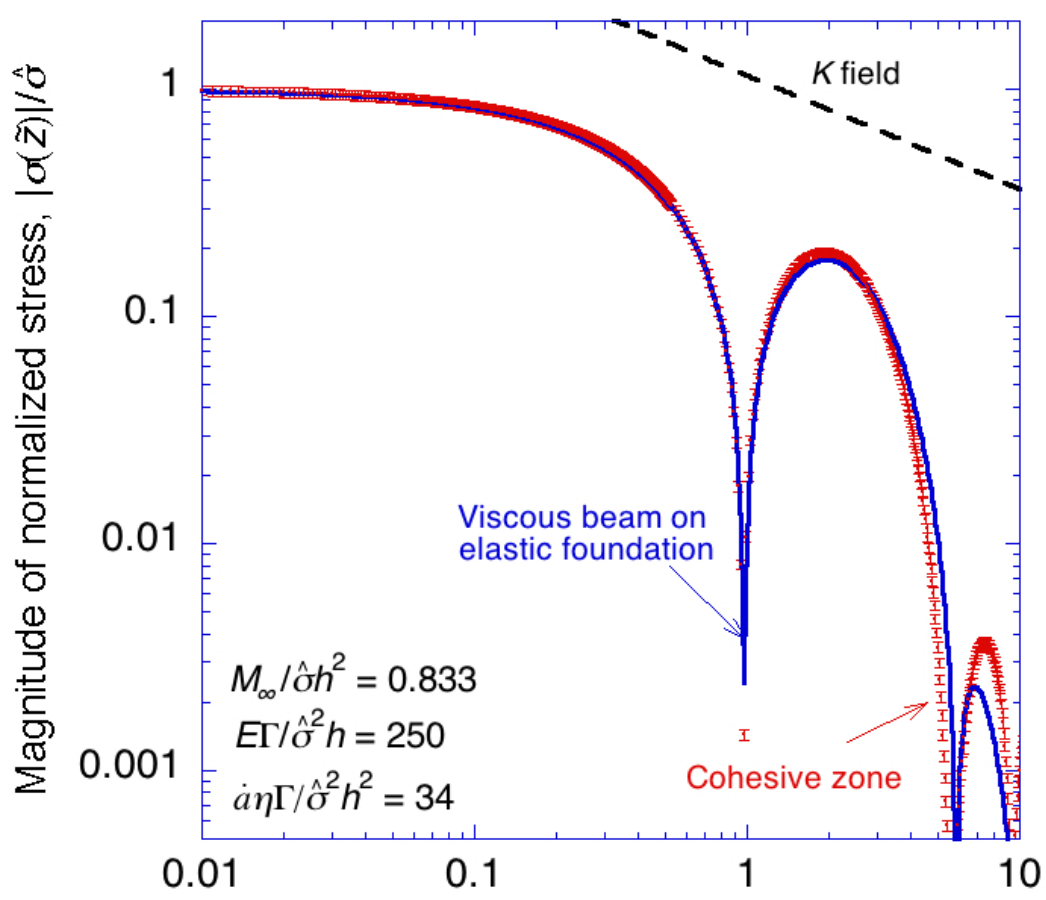

Normalized distance ahead of crack tip, $z / h$

Figure 6: Comparison between the stress distributions ahead of a steady-state crack for a viscous double-cantilever beam using a cohesive-zone models and an elastic foundation model for a small and large cohesive-length scale. 


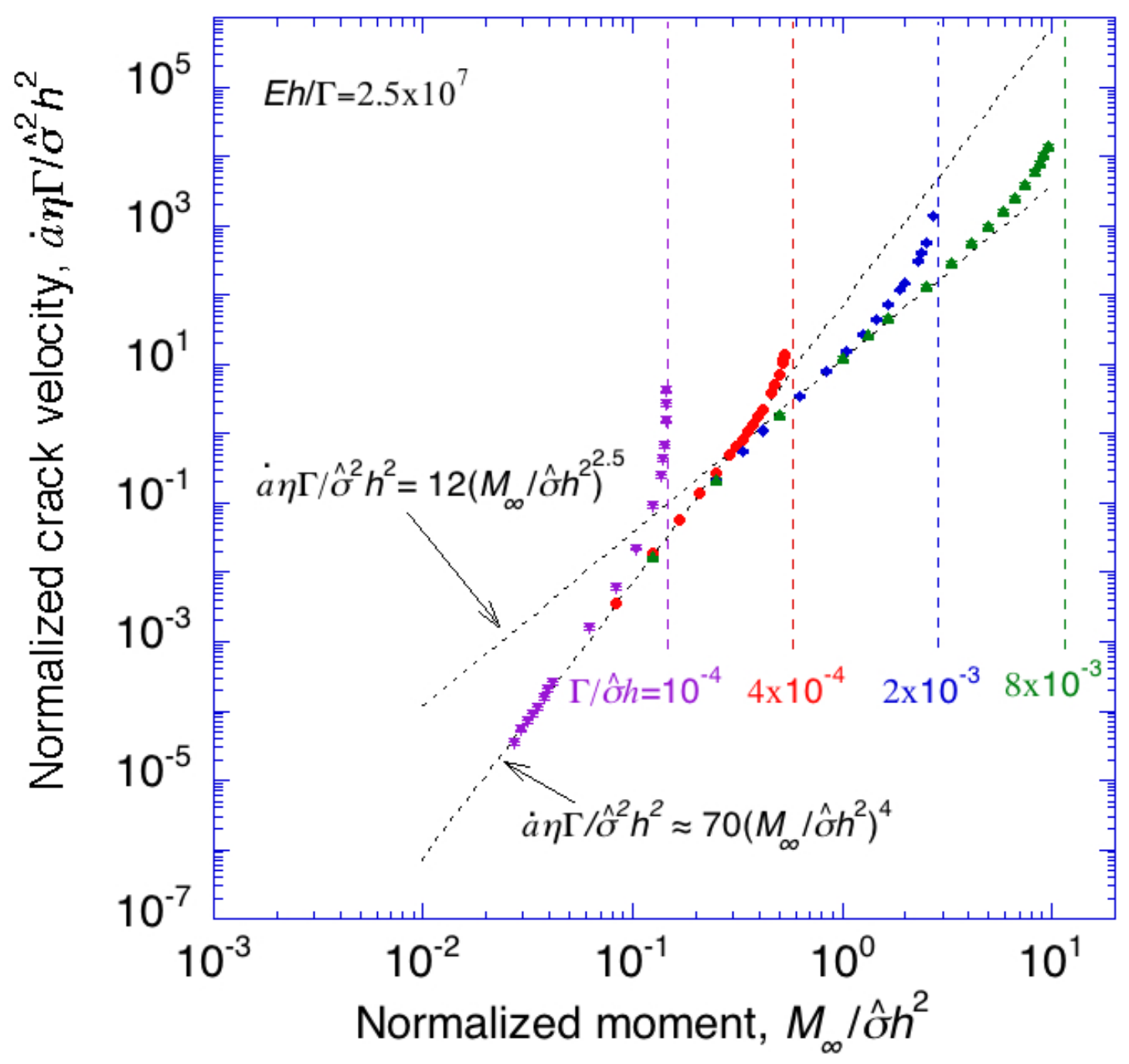

Figure 7: A summary of the three different crack-growth regimes, showing elastic fracture, crack growth at large viscous fracture lengths, and crack growth at small viscous fracture lengths. The crack velocity increases significantly as the applied moment approaches the critical moment for elastic fracture. 


\section{Nomenclature}

E Young's modulus

$\Gamma \quad$ Toughness of inetrface

$h \quad$ Thickness of beams

$\hat{\sigma} \quad$ Cohesive strength of interface

$M_{\infty} \quad$ Applied moment

$T_{n} \quad$ Normal traction

$k \quad$ Spring constant

$v \quad$ Displacement

$\tilde{v} \quad$ Non-dimensional displacement, $v / h$

$z \quad$ Distance ahead of crack tip

$\tilde{z} \quad$ Non-dimensional distance ahead of crack tip

$\beta_{o} \quad(3 k h / E)^{1 / 4}$

$\zeta \quad$ Elastic fracture length

$\tilde{\zeta} \quad$ Elastic fracture length scale, $\zeta / h$

$\eta \quad$ Uniaxial viscosity

$t$ time

$\dot{a} \quad$ crack velocity

$\lambda_{o} \quad\left(12 k h^{2} \eta \dot{a}\right)^{1 / 5}$

$\zeta_{v} \quad$ Viscous fracture-length scale

$\tilde{\zeta}_{v} \quad$ Non-dimensional viscous fracture-length scale

$\dot{\varepsilon}_{i j} \quad$ Strain-rate tensor

$v \quad$ Poisson's ratio

$\sigma_{i j}^{\prime} \quad$ Deviatoric stress tensor

$C^{*} \quad K^{2} / \eta$ where $K$ is the stress-intensity factor 